\title{
The self-choice effect from a multiple-cue perspective
}

\author{
TOMOYUKI WATANABE \\ Sendai Shirayuri Women's College, Sendai, Japan \\ and \\ SAL A. SORACI \\ Tufts University, Medford, Massachusetts
}

\begin{abstract}
The self-choice effectrefers to the fact that self-chosen items are rememberedbetter than experimenterassigned items (Takahashi, 1991). The present study investigated the hypothesis that (a) response choice involves relational processing as activation of both target and context items, and (b) such activated context items are effective as potential retrieval cues for recall of target items. In the experiment, participants chose (choice condition) or were assigned (force condition) a target to remember for each trial. Prior to free recall of the target items, context words, related new words, or unrelated words were presented in a recognition task as potential retrieval cues. The results of a subsequent free recall test indicated that the incidental cues were more effective in the choice condition than in the force condition. Also, recognition resulted in a greater rate of successfully recognized context words at the cost of increasing falsely recognized related new words in the choice condition in comparison with the force condition. These results indicated that response choice activates context items at encoding, which operate as potential retrieval cues for recall of target items. Such cuing mechanisms operative in the self-choice effect are consistent with the multiple-cue theory proposed by Soraci et al. (1994; see also Soraci et al., 1999) for generative processing.
\end{abstract}

The self-choice effect refers to the phenomenon that self-chosen items are remembered better than experimenterassigned items (Takahashi, 1991). In a typical study, participants are provided with several response candidates (e.g., COTTON: WOOL: SILK), and asked to choose one to remember. In one condition (force condition), one of the candidates is circled as a target (e.g., the word wool is circled, and the participants have to learn it), whereas in another condition (choice condition), participants can freely select a target (e.g., a participant chooses wool to remember). In a subsequent test phase, retention of the target items is better when participants have chosen targets, in comparison with the condition in which targets have been circled in advance. The phenomenon itself seems obvious, but its underlying mechanism remains undetermined. To address this issue, in the present study we examine the cognitive processes underlying this memory effect, specifically in terms of relational processing.

This article is dedicated to the memory of Sal A. Soraci, who passed way in August 2003. It is a revision of a part of a dissertation completed by the first author under the guidance of Dr. Soraci at Tufts University. A part of this research was presented at the 41st Annual Meeting of the Psychonomic Society, New Orleans. The authors thank Richard Chechile, Mike Carlin, Mike Toglia, Jeff Neuschatz, and several anonymous reviewers for their helpful comments on earlier versions of this paper. Correspondence may be sent to T. Watanabe, Department of Human Developmental Science, Sendai Shirayuri Women's College, 6-1 Honda-cho, Izumi-ku, Sendai, Miyagi 981-3107, Japan (e-mail: watanabe@ sendaishirayuri. ac.jp).
Many readers may notice that the self-choice effect is analogous to the generation effect (Slamecka \& Graf, 1978), which involves a retention advantage for active item generation relative to passive item reading. It is our view that the generation and the self-choice effects have much in common. As Begg, Snider, Foley, and Goddard (1989) speculated, item generation involves two processes: the production of response candidates, and the selection of a target. Because response candidates are provided in the case of choice, the latter process, the selection of a target, is a central process in the self-choice effect. Yet in spite of such commonality between the two active encoding phenomena, the self-choice effect has drawn little attention, and its underlying mechanism is therefore still unclear. Because of its potential contributions to theoretical developments in research on generative encoding as well as to educational applications (cf. Cordova \& Lepper, 1996; Toyota \& Tsujimura, 2000), it is important to investigate the self-choice effect's underlying processes.

Two prevailing accounts of the self-choice effect have been proposed, but they are rather questionable. One account argues that the self-choice effect results from enhanced motivation (Perlmuter, Monty, \& Kimble, 1971). It is assumed that choice allows participants to "perceive" control and motivates them to be engaged in the task (Monty, Geller, Savage, \& Perlmuter, 1979). Yet this is not a direct account of how choice processes enhance memory; it does not provide a delineation of the mechanisms incurred during response choice and the retrieval benefit. To illustrate 
those mechanisms, it is critical to address the cognitive processes involved.

The second account is an attempt to explain the selfchoice effect from the perspective of metamemory judgment. The assumption is that participants can choose "easier" items in the choice condition, which causes the self-choice effect (Takahashi, 1991, 1992). Two clear counterexamples have been reported, however. First, selfchoice effects were obtained not only with target items (e.g., wOOL), but also with context items (e.g., COTTON and SILK). If participants simply choose easier items, context items should be harder to remember, resulting in a negative self-choice effect for context items. The data indicated, however, that context items were remembered better in the choice condition than in the force condition (Monty, Perlmuter, Libon, \& Bennet, 1982; Perlmuter \& Monty, 1982; Watanabe, 2001). Second, it has been reported in several studies that response choice enhanced memory even when choice was fully "constrained." Previous studies, including those supporting the metamemory account, had employed "free" choice contexts, which allow participants to choose any items they like. However, Watanabe (2001) reported that the choice of a "correct" answer from a set of response candidates (similar to multiple-choice questions in an exam) resulted in better memory than did forced items, in a fully constrained encoding context. In his study, participants were provided with three response candidates and a phrase or sentence determining one of them (e.g., COTTON: WOOL: SILK followed by Cloth made from sheep), and chose or were assigned a correct response (i.e., WOOL). In this case, there would have been no opportunity for participants to choose easier items, but the effect was still obtained in the recognition test. These two counterexamples point strongly to the need for another cognitive account of the effect.

Along these lines, Takahashi (1997) discussed the role of distinctiveness in the self-choice effect. When a participant chooses one among several response candidates, she/he has to discriminate the chosen one from the others. As a result, it is reasonable to assume that the selected item is encoded with distinctiveness information (cf. Jacoby, Craik, \& Begg, 1979). However, it should be noted that item discrimination is not the only critical cognitive operation involved in response choice; item comparison is also an important process operating in choice. When participants compare response candidates, those candidates would be expected to be encoded together and should form a shared representation (cf. Logan \& Etherton, 1994). Item comparison essentially involves relational processing, because it focuses on relations among response candidates. Such "relatedness checking" could contribute to a subsequent retention advantage (cf. Donaldson \& Bass, 1980).

From this perspective, Watanabe (2001) recently proposed an account of the self-choice effect based on the multiple-cue hypothesis for the generation effect (Soraci et al., 1999; Soraci et al., 1994). The original multiple-cue hypothesis for the generation effect predicts that item gen- eration facilitates the activation of additional response candidates, which provide additional retrieval routes in subsequent remembering. To examine this hypothesis, Experiment 5 of Soraci et al. (1994) provided encoding contexts in which additional response candidates could be activated. The participants were required to complete word fragments that had three possible solutions (e.g., C_P), or they were simply presented with complete words. Each of the fragments or words was followed by a set of one or two simple cues. In one condition (CSS), only one positive cuing was provided (e.g., a policeman). In another condition (ISS), the cuing involved the rejection of one possible solution (e.g., NOT a policeman). In the other condition (IDS), two negative cues involved the rejection of two possible solutions (e.g., NOT a policeman; NOT a hat). The results indicated that recall was superior in the IDS (multiplecue) generate condition and that the generation effect was largest for the IDS conditions. This finding indicates that item generation at encoding facilitates the efficacy of cuing on subsequent memory tests.

Watanabe (2001) applied this hypothesis to the selfchoice effect and argued that choice requires participants to pay attention to context items (as well as target items) at encoding, and that the additional activation of context items involves the activation of potential retrieval cues for subsequent retrieval. Such a benefit is unlikely in the force condition, because item comparison is not required in this condition, and context items therefore draw little attention. He demonstrated the self-choice effect for context items, and also found that choice induced more false recognition when filler items were new words related to learned response candidates. However, this is still rather indirect evidence to conclude definitively that the multiple-cue process underlies the self-choice effect, because it did not include an examination of retrieval processes. The present study provides substantially more direct empirical support for the multiple-cue account by demonstrating not only that choice activates context items, but also that the activated context items function as retrieval cues.

In the present study, the "constrained" choice paradigm was employed in order to eliminate the item-selection factors. The self-choice effect in free recall was examined. Instead of the standard free recall paradigm, the recall test was administered after a recognition test. In the recognition task, target items were never presented. The participants were required to make recognition judgments on context items. The main purpose of this recognition test was to provide some context items as incidental retrieval cues for subsequent free recall. Context words were presented for some targets but were not presented for other targets, and subsequent target recall was compared between conditions with and without prior exposure to the context words. In addition, the participants' performances on the recognition test were analyzed in order to examine memory for the context words.

If context items, as well as target items, are activated through choice (clearly a generative activity), recognition for context items should be better in the choice condition 
than in the force condition. Furthermore, because category members were utilized as a response set for choice, such activation of context items could involve category activation, concomitantly inducing false recognition of new category members. In summary, target recall should benefit from prior presentation of context words to a greater degree in the choice than in the force condition.

\section{METHOD}

\section{Participants}

Forty-nine undergraduate students participated in the experiment in order to fulfill partial requirements for an introductory psychology class.

\section{Materials}

Four words were selected from each of 36 categories out of Battig and Montague's (1969) 56 categories. For each of the categories, three of the four words formed a response set as response candidates for a choice/force trial, and the remaining word was reserved as a related lure item for the recognition task. An additional six new categories were also selected, with two members from each reserved as unrelated lure items for the recognition task. A target word in each of the response sets was determined in advance, and a cue sentence was generated so as to distinguish the target from the other two candidates in each of the response sets. Six types of printed lists were constructed for counterbalancing, including 36 choice/force trials and 48 recognition items. Half of the learning trials were choice and the others were force. The 48 recognition items were composed of 24 context words (i.e., 12 pairs of context items in each encoding condition) as old items for the recognition task, and 12 related lures and 12 unrelated lures as new items. Those 2 (choice/force) $\times 3$ (type of recognition items) conditions were counterbalanced across the types of the lists. The orders of response sets and the recognition items were randomized respectively per list type. Arithmetic problems were prepared to serve as an interpolated task between the learning and the recognition phases. All of these forms, written instructions for each phase, and a blank sheet of paper for free recall were bound into a booklet in the appropriate order.

\section{Procedures}

The experiment comprised four phases: learning, interpolated task, recognition, and free recall. In the learning phase, the participants were presented with 36 choice/force trials, each of which consisted of three response candidates and a cue sentence determining a single item from the candidates. When one of the candidates was circled, it always matched the cue sentence, and the participants were forced to choose it and write it down in a blank next to the sentence (force condition). Otherwise, the participants were required to choose a correct response and write it down (choice condition). The participants were allowed $10 \mathrm{sec}$ for completing each of the trials. At the beginning of this phase, the participants were instructed to remember items for later memory tests, but details about the tests were not mentioned.

After the learning phase, the participants were engaged in a 3-min interpolated task, in which they were asked to solve arithmetic problems. Next, in the recognition phase, the participants were provided with a 48-word list and asked to circle any words that they encountered in the learning phase. Three different types of words were presented in this phase, to provide incidental cues for subsequent free recall. The first type was an old-cue, which was the same as a context word presented in the learning and an old item in the recognition task (e.g., СотTON). The second was a related-cue, a new word related to a response set in the learning (e.g., NYLON). The last type was an unrelated-cue, which had no relation to the presented words in the learning (e.g., CAR). Three minutes were given for this task. Fi- nally, participants engaged in a 5-min free recall test, in which they were instructed to recall as many of the words they had written down in the learning phase (i.e., target items) as possible.

\section{Design and Analysis}

A 2 (encoding task) $\times 3$ (cue type) within-participants design was employed. Analyses were conducted for recall performance, with proportions of recalled items as the dependent variable. For the recognition task, hit rates of context items were compared between the learning conditions. False alarm rates were also compared between choice and force for related lures, and between related and unrelated lures.

\section{RESULTS}

\section{Learning}

Mean percentages of correct responses at learning were $96.41 \%(S D=1.14)$ in the choice condition, and $99.65 \%$ $(S D=0.35)$ in the force condition. The following analyses were based on only the correct responses.

\section{Recognition}

As is indicated in Table 1, the context items were recognized better in the choice condition than in the force condition $\left[F(1,48)=58.068, M S_{\mathrm{e}}=.047, p<.001\right]$. However, the choice condition also produced significantly greater false memory. Table 1 also indicates a greater false alarm rate in the choice condition than in the force condition $\left[F(1,48)=8.871, M S_{\mathrm{e}}=.015, p=.005\right]$. In addition, related lure items (combining choice and force conditions) were more falsely recognized than were unrelated lures $\left[F(1,48)=57.950, M S_{\mathrm{e}}=.015, p<.001\right]$. The mean $d$ 's based on adjusted hit and false alarm rates (cf. Gallo, Roediger, \& McDermott, 2001) were 1.28 in the choice condition, and .59 in the force condition. The difference was significant $\left[F(1,48)=21.538, M S_{\mathrm{e}}=.538\right.$, $p<.001]$.

\section{Free recall}

Table 2 shows the results of free recall. Under the choice condition, planned contrasts indicated that words were recalled better when old-cues were presented in the prior recognition task than in the other two conditions combined $\left[F(1,48)=66.141, M S_{\mathrm{e}}=.040, p<.001\right]$, and that recall was better when related-cues were presented in advance of recall than when unrelated-cues were presented $\left[F(1,48)=31.707, M S_{\mathrm{e}}=.040, p<.001\right]$. Under the force condition, the old-cue condition was still higher in recall

Table 1

Mean Hit and False Alarm Rates by Encoding Task

\begin{tabular}{|c|c|c|c|c|c|c|}
\hline & \multicolumn{4}{|c|}{ Condition } & & \\
\hline & \multicolumn{2}{|c|}{ Choice } & \multicolumn{2}{|c|}{ Force } & \multicolumn{2}{|c|}{ Unrelated } \\
\hline & $M$ & $\overline{S D}$ & $M$ & $\overline{S D}$ & $M$ & $\overline{S D}$ \\
\hline \multicolumn{7}{|l|}{ Hits } \\
\hline Old-cue & .71 & .19 & .37 & .24 & & \\
\hline \multicolumn{7}{|l|}{ False Alarms } \\
\hline Related-cue & .21 & .19 & .14 & .16 & & \\
\hline Unrelated-cue & \multicolumn{2}{|c|}{ - } & \multicolumn{2}{|c|}{ - } & .02 & .04 \\
\hline
\end{tabular}


Table 2

Mean Recall Rate for Target Items by Encoding Task and Cue Type

\begin{tabular}{llllll}
\hline & \multicolumn{4}{c}{ Encoding Task } \\
\cline { 2 - 3 } \cline { 5 - 6 } Cue type & \multicolumn{2}{c}{ Choice } & & \multicolumn{2}{c}{ Force } \\
\cline { 2 - 5 } \cline { 5 - 6 } & $M$ & $S D$ & & $M$ & $S D$ \\
\hline Old-cue & .52 & .22 & .33 & .22 \\
Related-cue & .39 & .24 & .27 & .22 \\
Unrelated-cue & .23 & .18 & .23 & .20 \\
\hline
\end{tabular}

than in the other two conditions combined $[F(1,48)=$ $\left.11.289, M S_{\mathrm{e}}=.030, p=.002\right]$, but there was no significant difference between the related-and the unrelated-cue conditions $\left[F(1,48)=2.691, M S_{\mathrm{e}}=.030, p=.107\right]$. This interaction between the choice and the force conditions was confirmed in the supplementary omnibus analysis of variance $\left[F(2,96)=7.201, M S_{\mathrm{e}}=.038, p=.001\right]$. Selfchoice effects were obtained in the old- and related-cue conditions $\left[F(1,48)=23.288, M S_{\mathrm{e}}=.049, p<.001\right.$; $F(1,48)=8.325, M S_{\mathrm{e}}=.048, p=.006$, respectively], but not in the unrelated-cue condition $(F<1)$.

\section{DISCUSSION}

In agreement with previous studies (Monty et al., 1982; Perlmuter \& Monty, 1982; Watanabe, 2001), hit rates for context items were higher in the choice condition than in the force condition. These results support the view that choice induces activation of both target and context items. The self-choice effect for context items is thus in contradistinction to the account based on metamemory judgments, and indicates the critical role of context items in the self-choice effect.

This notion is further supported by the false alarm rates. As in recent studies that have resulted in findings of false memory (e.g., Roediger \& McDermott, 1995), the choice process induced greater false memory when participants were required to recognize all the items that they encountered at learning. It should be noted that the encoding activity involved in selecting responses enhanced memory for items, and it simultaneously induced greater false memory. Thus instantiates a "more is less" pattern, the phenomenon that remembering more items, in certain experimental contexts, leads to less accurate memory (cf. Toglia, Neuschatz, \& Goodwin, 1999). The greater false alarm rate in the choice condition indicated that the activation of target and context items expanded to a category representation, and therefore, this finding further supports the hypothesis that an entire response set is involved in the choice condition. Thus the relational or organizational processing of target and context items is induced as a result of response choice. Overall, this pattern of results cannot be interpreted simply in terms of the distinctiveness account.

The third and most important finding in the present study was that, when context items were provided as incidental cues, such cues facilitated subsequent target recall to a greater degree in the choice condition than in the force condition. The results indicated that context items operated as more effective retrieval cues in the choice condition. In addition, it was found that related new items that were assumed to be activated as a result of category activation were also more effective as retrieval cues in the choice condition. These results are consistent with Soraci et al.'s (1994; see also Soraci et al., 1999) multiple-cue account. Conceptually, the choice manipulationin the present study is directly analogous to the generation manipulationutilized by Soraci et al. (1994). Furthermore, the effect was diminished under the unrelated-cue condition, possibly due to some inhibitive mechanisms of unrelated cuing (cf. Nickerson, 1984). This fact raises the possibility that retrieval processes in the choice condition are more cue dependent, which would be consistent with the multiple-cue perspective.

Several possible alternative explanations for the present results should be addressed. One involves an attentionbased account, which assumes that choice essentially demands the participant's attention with respect to all response candidates. However, this simple account leads to contradictory predictions, in that spreading attention to all response candidates should reduce the allocation of attentional resources to the targets, resulting in worse memory. The account based on levels of processing (LOP; Craik \& Lockhart, 1972; Craik \& Tulving, 1975) also seems to be consistent with the self-choice effect. However, the LOP account does not address the retrieval component demonstrated in the present study. The LOP account is essentially an encoding-based account, but the present findings about cuing mechanisms demonstrate that important retrievalbased processes are responsible for the self-choice effect. Furthermore, the LOP account focuses on individual-item factors, but the present study has demonstrated a critical relational factor involved in the self-choice effect (cf. Einstein \& Hunt, 1980).

Fuzzy-trace theory (Reyna \& Brainerd, 1995) is more compatible with the present results. It assumes that memory is composed of two independent memory traces, verbatim and gist. In the case of the self-choice effect, and specifically in choice conditions, the encoding of the target item enhances verbatim memory, and the encoding of whole response sets creates gist memory. The remembering of target items can benefit from both types of memory, because such items can be encoded as members of response sets, as well as distinctive targets. This theory is consistent with the multiple-cue account, in that both emphasize the critical roles of context items in the self-choice effect. Fuzzy-trace theory is also relevant to the present results related to false recognition. Because participants were required to recognize context items, the recognition test was more dependent on gist memory. Of course, fuzzy-trace theory has usually been demonstrated in relatively passive encoding contexts in which "choice" or "generation" is not involved. However, it remains possible that there is an explanatory role for fuzzy-trace theory in supplementing the cuing framework in the present study.

The novel application of the multiple-cue perspective to the self-choice effect in the present study clarifies the 
relationship between generation and self-choice effects. The multiple-cue perspective was originally introduced for delineating the cognitive mechanisms underlying the generation effect (Soraci et al., 1994), and the present study appropriates it as a viable explanation for the self-choice effect. If similar cognitive processing underlies both the effects, multiple cuing can provide a foundation for discussing both phenomena in the same theoretical context.

\section{REFERENCES}

Battig, W. F., \& Montague, W. E. (1969). Category norms for verbal items in 56 categories: A replication and extension of the Connecticut category norms. Journal of Experimental Psychology, 80 (3, Pt. 2), 146.

BegG, I., Snider, A., Foley, F., \& Goddard, R. (1989). The generation effect is no artifact: Generating makes words distinctive. Journal of Experimental Psychology: Learning, Memory, \& Cognition, 15, 977 989.

CORDOVA, D. I., \& LEPPER, M. R. (1996). Intrinsic motivation and the process of learning: Beneficial effects of contextualization, personalization, and choice. Journal of Educational Psychology, 88, 715-730.

CRAIK, F. I. M., \& LockHART, R. S. (1972). Levels of processing: A framework for memory research. Journal of Verbal Learning \& Verbal Behavior, 11, 671-684.

Craik, F. I. M., \& Tulving, E. (1975). Depth of processing and the retention of words in episodic memory. Journal of Experimental Psychology: General, 104, 268-294.

DONALDSON, W., \& BASs, M. (1980). Relational information and memory for problem solutions. Journal of Verbal Learning \& Verbal Behavior, 19, 26-35.

EINSTEIN, G. O., \& HUNT, R. R. (1980). Levels of processing and organization: Additive effects of individual item and relational processing. Journal of Experimental Psychology: Human Learning \& Memory, 6, 588-598.

Gallo, D. A., Roediger, H. L., III, \& McDermott, K. B. (2001). Associative false recognition occurs without strategic criterion shifts. Psychonomic Bulletin \& Review, 8, 579-586.

JACOBY, L. L., CRAIK, F. I. M., \& BEGG, I. (1979). Effects of decision difficulty on recognition and recall. Journal of Verbal Learning \& Verbal Behavior, 18, 585-600.

LogAn, G. D., \& Etherton, J. L. (1994). What is learned during automatization?: The role of attention in constructing an instance. Journal of Experimental Psychology: Learning, Memory, \& Cognition, 20, 1022-1050.

Monty, R. A., Geller, E. S., Savage, R. E., \& Perlmuter, L. C. (1979). The freedom to choose is not always so choice. Journal of Experimental Psychology: Human Learning \& Memory, 5, 170-178.
Monty, R. A., Perlmuter, R. C., Libon, D., \& Bennet, T. (1982). More on contextual effects on learning and memory. Bulletin of the Psychonomic Society, 20, 293-296.

NiCKERSON, R. S. (1984). Retrieval inhibition from part-set cuing: A persisting enigma in memory research. Memory \& Cognition, 12, 531552.

Perlmuter, L. C., \& Monty, R. A. (1982). Contextual effects on learning and memory. Bulletin of the Psychonomic Society, 20, 290-292.

Perlmuter, L. C., Monty, R. A., \& Kimble, G. A. (1971). Effect of choice on paired-associate learning. Journal of Experimental Psychology, 91, 47-53.

REYNA, V. F., \& BRAINERd, C. J. (1995). Fuzzy-trace theory: An interim synthesis. Learning \& Individual Differences, 7, 1-75.

Roediger, H. L., III, \& MCDERMOTt, K. B. (1995). Creating false memories: Remembering words not presented in lists. Journal of Experimental Psychology: Learning, Memory, \& Cognition, 21, 803-814.

SLAMECKA, N. J., \& GRAF, P. (1978). The generation effect: Delineation of a phenomenon. Journal of Experimental Psychology: Human Learning \& Memory, 4, 592-604.

Soraci, S. A., Carlin, M. T., Chechile, R. A., Franks, J. J., Wills, T., $\&$ WATANABE, T. (1999). Encoding variability and cuing in generative processing. Journal of Memory \& Language, 41, 541-559.

Soraci, S. A., Franks, J. J., Bransford, J. D., CheChile, R. A., Belli, R. F., CARR, M., \& CARLIN, M. [T.] (1994). Incongruous item generation effects: A multiple-cue perspective. Journal of Experimental Psychology: Learning, Memory, \& Cognition, 20, 67-78.

TAKAHASHI, M. (1991). The role of choice in memory as a function of age: Support for a metamemory interpretation of the self-choice effect. Psychologia, 34, 254-258.

TAKAHASHI, M. (1992). Memorial consequences of choosing nonwords: Implication for interpretations of the self-choice effect. Japanese Psychological Research, 34, 35-38.

TAKAHASHI, M. (1997). Kioku ni okeru fugoka horyaku no kenkyu [Studies of encoding strategies in memory]. Kyoto, Kitaoji-shobo. (In Japanese)

Toglia, M. P., Neuschatz, J. S., \& Goodwin, K. A. (1999). Recall accuracy and illusory memories: When more is less. Memory, 7, 233256.

Toyota, H., \& Tsujimura, M. (2000). The self-choice elaboration effects on incidental memory of Japanese historical facts. Perceptual \& Motor Skills, 91, 69-78.

WATANABE, T. (2001). Effects of constrained choice on memory: The extension of the multiple-cue hypothesis to the self-choice effect. Japanese Psychological Research, 43, 98-103.

(Manuscript received November 17, 2002; revision accepted for publication December 5, 2002.) 